\title{
Infiltração de 5-fluorouracil no pré-operatório do pterígio
}

Preoperative 5-fluorouracil infiltration in pterygium surgery

Claudia Akemi Shiratori 1

Érika Hoyama ${ }^{1}$

Silvana Artioli Schellini ${ }^{2}$

Carlos Roberto Padovani ${ }^{3}$

\section{RESUMO}

Objetivo: Avaliar o efeito do 5-fluorouracil (5FU) injetado intralesionalmente na cabeça do pterígio no período pré-operatório. Métodos: Foram estudados 53 olhos (52 pacientes), sendo 28 pterígios primários e 25 recidivados, divididos em dois grupos: grupo 1 (G1), composto por indivíduos que receberam a injeção de 5-fluorouracil 30 dias antes do procedimento cirúrgico e grupo 2 (G2), no qual o 5-fluorouracil foi injetado 10 dias antes da cirurgia. Todas as cirurgias foram realizadas seguindo-se a mesma técnica cirúrgica, pelo mesmo cirurgião. Os pacientes foram reavaliados 7 , 30 e 60 dias após a cirurgia. Os resultados observados foram submetidos à análise estatística. Resultados: A amostra estudada foi constituída por $52,8 \%$ de pterígios primários e $47,2 \%$ de recidivados, sendo composta igualmente por indivíduos de ambos os sexos. Não ocorreram complicações decorrentes da infiltração da droga. A recidiva foi mais freqüente nos pterígios recidivados e no G1. Conclusão: $\mathrm{O}$ uso intralesional de 5-fluorouracil no pré-operatório do pterígio não provocou efeitos deletérios aos olhos estudados. Houve menor recorrência quando usado o 5 -fluorouracil 10 dias antes da exérese cirúrgica, em relação à aplicação 30 dias antes do procedimento.

Descritores: Pterígio/cirurgia; Soluções oftálmicas; Fluorouracil/administração \& dosagem; Fluorouracil/uso terapêutico; Mitomicina/uso terapêutico; Recidiva/prevenção \& controle; Cuidados intra-operatórios

INTRODUÇÃO

Pterígio é uma doença ocular benigna, caracterizada por proliferação conjuntival que se estende sobre a córnea. A sua patogênese ainda não completamente esclarecida, associada a uma variável taxa de recidiva, constitui um desafio para o médico oftalmologista no que se refere à escolha da melhor opção terapêutica.

Uma das modalidades de tratamento coadjuvante é o uso de drogas antimitóticas, empregadas desde a década de 60 , como o Thio-tepa e a Mitomicina C.

O uso do Thio-tepa provocava alterações transitórias nos tecidos oculares (como diminuição da sensibilidade corneana, alteração do volume e estabilidade do filme lacrimal e da vitalidade dos epitélios corneano e conjuntival) $^{(1)} \mathrm{e}$ até déficit visual permanente ${ }^{(2)}$, o que abriu espaço para a Mitomicina C, que, na década de 80 , surgiu como uma droga promissora para o tratamento coadjuvante do pterígio, sendo amplamente estudada e usada com bons resultados quanto à diminuição dos índices de recidiva de pterígios primários e recidivados ${ }^{(3-5)}$.

Contudo, há também inúmeros relatos de complicações freqüentes e importantes associadas ao uso da Mitomicina $\mathrm{C}$, como: glaucoma secundário, edema e perfuração corneana, corectopia, irite, catarata, calcificação escle- 
ral, fotofobia, dor incapacitante ${ }^{(6-8)}$, além de defeito epitelial persistente ${ }^{(9)}$. Os efeitos colaterais podem surgir vários anos após a aplicação.

Até o presente, pouco se conhece sobre o uso do 5-fluorouracil $(5 \mathrm{FU})$ como tratamento coadjuvante do pterígio. Assim como a Mitomicina $\mathrm{C}$, sua ação anti-fibroblástica ocorre pela inibição do DNA, porém, por mecanismos diferentes; a citotoxicidade e a inibição da proliferação de fibroblastos da cápsula de Tenon são menores com o $5 \mathrm{FU}^{(10)}$, o que o torna uma droga mais segura.

Estudos experimentais mostraram que o $5 \mathrm{FU}$ não provoca alterações deletérias aos tecidos oculares, quando utilizado em aplicação tópica ${ }^{(11)}$, ou subconjuntival ${ }^{(12)}$.

O 5FU, em uso tópico no intra-operatório, não causou as complicações relatadas com a Mitomicina C, observando-se redução da taxa de recidiva ${ }^{(13)}$.

Recentemente foi sugerido o uso da Mitomicina C em infiltração intra-lesional, na cabeça do pterígio, 30 dias antes da cirurgia ${ }^{(14)}$.

Entretanto, o 5FU foi usado de modo injetável apenas no período pós-operatório, utilizado em 4 infiltrações subconjuntivais ${ }^{(15)}$. Não existe, até o momento, relato de infiltração do $5 \mathrm{FU}$ no pré-operatório, como tratamento coadjuvante do pterígio.

$\mathrm{O}$ presente estudo tem por objetivo avaliar o uso do $5 \mathrm{FU}$ aplicado sob a forma de infiltração intra-lesional na cabeça do pterígio no período pré-operatório.

\section{MÉTODOS}

Este foi um estudo prospectivo e incluiu 53 olhos de 52 portadores de pterígios primários e recidivados, atendidos consecutivamente no Hospital das Clínicas da UNESP, Botucatu - SP, no período de novembro de 2000 a maio de 2001.

A classificação dos pterígios foi feita por um mesmo examinador, de acordo com a padronização usada no serviço, baseada na observação clínica das seguintes características:

1. Primário ou recidivado

2. Aspecto da proliferação fibrovascular:

a) Involutivo: aspecto atrófico, visibilização dos vasos episclerais no limbo

b) Carnoso: aspecto espessado, impossibilitando observar vasos episclerais

3. De acordo com a extensão sobre a superfície corneana:

a) Grau 1: atinge o limbo em até $2 \mathrm{~mm}$

b) Grau 2: atinge a borda pupilar

c) Grau 3: ultrapassa a borda pupilar, atingindo o eixo visual

Todos os pacientes foram submetidos à injeção de $0,2 \mathrm{ml}$ de $5 \mathrm{FU}$ (Roche $25 \mathrm{mg} / \mathrm{ml}$ ) intra-lesional na cabeça do pterígio, feita após a instilação tópica de cloridrato de proximetacaína (Allergan), usando uma seringa de $1 \mathrm{ml}$ e agulha 13,0 X 4,5.

Os pacientes foram divididos aleatoriamente em 2 grupos (G1 e G2), de forma que a injeção de 5FU foi feita 30 dias e 10 dias antes do procedimento cirúrgico, respectivamente.
Todas as cirurgias foram realizadas pelo mesmo cirurgião e a mesma equipe de auxiliares, empregando-se a técnica do retalho conjuntival, iniciando-se pela anestesia tópica com cloridrato de proximetacaína (Allergan) e subconjuntival com lidocaína $2 \%$ com vasoconstritor (Astra). Procedeu-se a delaminação corneana com bisturi lâmina 15 , exérese da cabeça do pterígio com tesoura e ressecção da Tenon adjacente. Foi realizado deslizamento de retalho superior e inferior para fe- chamento da conjuntiva próxima, na região do limbo, usando fio trançado absorvível sintético 7-0 (Vicryl - Ethicon), em dois pontos separados, recobrindo-se a esclera desnuda pela remoção da lesão. Ao final da cirurgia, foram aplicados colírio de cloranfenicol e dexametasona (Alcon) e pomada de ácido retinóico, cloranfenicol e vitamina A (Allergan). O curativo oclusivo foi mantido por 24 horas e retirado pelo próprio paciente, no domicílio. Durante o pós-operatório foi prescrito colírio de cloranfenicol e dexametasona (Alcon) de $4 / 4$ horas por 7 dias e de $6 / 6$ horas até 30 dias, quando então, a medicação era suspensa.

Após a cirurgia, os pacientes foram avaliados no $7^{\circ}, 30^{\circ} \mathrm{e}$ $60^{\circ}$ dias, observando-se as queixas inicial e final (hiperemia e ardência, referidas pelo paciente) e, ao exame biomicroscópico, o aspecto da região operada (em relação à presença de hiperemia, ou outras possíveis alterações, por avaliação qualitativa subjetiva) e a presença de complicações. Considerou-se como recidiva o reaparecimento de proliferação fibrovascular sobre a córnea.

Os dados relativos à distribuição do pterígio segundo grupo e sexo, grupo e ocorrência de recidiva foram submetidos à análise estatística pelo método de Goodman para contrastes entre e dentro de populações binomiais ${ }^{(16-17)}$. A significância do teste está apontada nas tabelas por meio de letras maiúsculas ou minúsculas, de forma que duas letras diferentes indicam diferença estatística significativa $(p<0,05)$. As letras minúsculas foram utilizadas para comparação de grupos (G1 X G2), dispostas no sentido vertical e as maiúsculas na comparação das categorias de respostas (tipo de pterígio e ocorrência de recidiva), indicadas no sentido horizontal.

\section{RESULTADOS}

Dos 53 olhos que compuseram a amostra do estudo, $52,8 \%$ eram pterígios primários e $47,2 \%$, recidivados; o G1 (injeção 30 dias antes do procedimento) foi composto predominantemente por pterígios primários $(68,0 \%)$ e o G2 (injeção 10 dias antes do procedimento), por pterígios recidivados $(60,7 \%)$ (Tabela 1$)$.

No G1, 64,7\% dos portadores de pterígio primário eram do sexo masculino, havendo predomínio do sexo feminino dentre os recidivados (Tabela 1). No G2, a distribuição por sexo foi semelhante entre os pterígios primários, havendo predomínio do sexo feminino nos pterígios recidivados (Tabela 1).

A injeção de 5FU foi realizada sem intercorrências em todos os pacientes, e não se observou qualquer alteração no período que transcorreu entre a injeção até o dia da cirurgia.

Com relação à idade, dentre os pterígios primários, a mediana 


\begin{tabular}{|c|c|c|c|c|c|c|}
\hline Grupo & Sexo & Primário & & Recidivado & & Total \\
\hline \multirow[t]{2}{*}{$\mathrm{G} 1$} & Feminino & $6(35,3 \%)$ & $\mathrm{a}$ & $5(62,5 \%)$ & $b$ & 11 \\
\hline & Masculino & $11(64,7 \%)$ & $b$ & $3(37,5 \%)$ & $\mathrm{a}$ & 14 \\
\hline \multirow[t]{2}{*}{ G 2} & Feminino & $6(54,5 \%)$ & a & $13(76,5 \%)$ & $b$ & 19 \\
\hline & Masculino & $5(45,5 \%)$ & $a$ & $4(23,5 \%)$ & $\mathrm{a}$ & 9 \\
\hline G 1 & & $17(68,0 \%)$ & B & $8(32,0 \%)$ & $A$ & 25 \\
\hline G 2 & & $11(39,3 \%)$ & A & $17(60,7 \%)$ & B & 28 \\
\hline Total & & $28(52,8 \%)$ & & $25(47,2 \%)$ & & 53 \\
\hline
\end{tabular}

foi de 48 anos no G1 e 49 no G2; dentre os recidivados, 61 anos para o G1 e 50 para o G2 (Tabela 2).

No G1, os pterígios eram do tipo carnoso em $35,3 \%$ e involutivos em $64,7 \%$, sendo $90,9 \%$ de grau 2 . No G2, $54,5 \%$ eram carnosos e $45,5 \%$ involutivos, sendo $82,3 \%$ de grau 2 .

Após a cirurgia de exérese do pterígio, as alterações oculares observadas foram: hiperemia, deiscência da sutura conjuntival (afastamento dos bordos da incisão, permitindo a visibilização da esclera devido à soltura do ponto), ceratite e cisto conjuntival com "dellen" corneano.

A hiperemia conjuntival ocorreu em todos os pacientes no $7^{\circ} \mathrm{PO}$, persistindo em alguns portadores de pterígios primários, assim como de recidivados, até o $60^{\circ} \mathrm{PO}$, tanto em G1, como em G2. (Tabela 3)

Os casos de deiscência da sutura conjuntival, todos observados no $7^{\circ} \mathrm{PO}$, ocorreram mais freqüentemente nos indivíduos do G1 (24,0\% em primários e 32,0\% em recidivados). No G2, ocorreram em 10,8\% (7,1\% em primários e 3,6\% em recidivados) (Tabela 4 ).

No G2, ocorreu 1 caso de ceratite, observado no $7^{\circ} \mathrm{PO}$, em uma paciente do sexo feminino, com pterígio primário involutivo

\begin{tabular}{|c|c|c|c|c|}
\hline \multirow[t]{2}{*}{ Grupo } & & \multicolumn{3}{|c|}{ Idade } \\
\hline & & Mínima & Mediana & Máxima \\
\hline \multirow[t]{2}{*}{$P$} & G 1 & 29 & 48 & 76 \\
\hline & G 2 & 24 & 49 & 67 \\
\hline \multirow[t]{2}{*}{$\mathrm{R}$} & G 1 & 41 & 61 & 78 \\
\hline & G 2 & 28 & 50 & 71 \\
\hline $\mathrm{P}=$ primá & $=$ rec & & & \\
\hline
\end{tabular}

de grau 2, que foi tratada com colírio de lágrima artificial, com melhora. Um dos pacientes evoluiu no $30^{\circ} \mathrm{PO}$ com cisto conjuntival na área da excisão, associado a "dellen" corneano, sendo submetido à exérese do cisto.

$\mathrm{O}$ índice de recidiva foi maior no grupo G1, tanto para pterígios primários, como recidivados. Assim, nos pterígios primários a recidiva ocorreu em 52,9\% do G1 e em $27,3 \%$ do G2, com diferença estatística significativa (Tabela 5).

Considerando-se os pterígios recidivados também houve diferença significativa, sendo de 75,0\% no G1 e 47,1\% no G2 (Tabela 5).

Dentre os 26 casos que tiveram recidiva, $26,7 \%$ tiveram deiscência de sutura no $7^{\circ} \mathrm{PO}$ ( 2 casos de pterígio primário e 2 , recidivados).

Não foi observada nenhuma outra complicação decorrente do uso do $5 \mathrm{FU}$ pré-operatório, usado sob a forma de infiltração na cabeça do pterígio.

DISCUSSÃO

As drogas antimetabólicas surgiram como uma nova proposta para o tratamento adjuvante do pterígio e também do glaucoma, devido à sua ação anti-fibroblástica.

Schellini et al. ${ }^{(13)}$ utilizaram o 5FU tópico, aplicado no intraoperatório do pterígio, não observando efeitos deletérios para o olho. Além deste estudo, os estudos experimentais ${ }^{(11-12)}$ nos incentivaram a utilizar a droga em infiltração subconjuntival na cabeça do pterígio previamente à cirurgia.

Este tipo de infiltração intra-lesional pré-operatória já havia sido previamente sugerida utilizando-se a Mitomicina $\mathrm{C}^{(14)}$.

A amostra do presente estudo foi constituída por indivíduos portadores de pterígio primário e recidivado, de ambos os sexos, sem diferença estatisticamente significativa.

\begin{tabular}{|c|c|c|c|c|}
\hline Hiperemia & P 0 & Pterígios primários & Pterígios recidivados & Total \\
\hline \multirow[t]{3}{*}{ G 1} & $7^{0}$ & $17(100,0 \%)$ & $8(100,0 \%)$ & $25(100,0 \%)$ \\
\hline & $30^{\circ}$ & $11(64,7 \%)$ & $6(75,0 \%)$ & $17(70,6 \%)$ \\
\hline & $60^{\circ}$ & $6(35,3 \%)$ & $5 \quad(62,5 \%)$ & $11(44,0 \%)$ \\
\hline \multirow[t]{3}{*}{ G 2} & $7^{\circ}$ & $11(100,0 \%)$ & $17(100,0 \%)$ & $28(100,0 \%)$ \\
\hline & $30^{\circ}$ & $8(72,7 \%)$ & $7 \quad(41,2 \%)$ & $15(60,7 \%)$ \\
\hline & $60^{\circ}$ & $7 \quad(63,6 \%)$ & $7 \quad(41,2 \%)$ & $14(50,0 \%)$ \\
\hline
\end{tabular}




\begin{tabular}{|c|c|c|c|c|}
\hline Deiscência & P O & Pterígios primários & Pterígios recidivados & Total \\
\hline \multirow[t]{3}{*}{ G 1} & $7^{\circ}$ & $6(24,0 \%)$ & $8(32,0 \%)$ & $14(56,0 \%)$ \\
\hline & $30^{\circ}$ & $0 \quad(0,0 \%)$ & $0 \quad(0,0 \%)$ & $0(0,0 \%)$ \\
\hline & $60^{\circ}$ & $0 \quad(0,0 \%)$ & $0 \quad(0,0 \%)$ & $0(0,0 \%)$ \\
\hline \multirow[t]{3}{*}{ G 2} & $7^{\circ}$ & $2(7,2 \%)$ & $1 \quad(3,6 \%)$ & $3(10,8 \%)$ \\
\hline & $30^{\circ}$ & $0 \quad(0,0 \%)$ & $0 \quad(0,0 \%)$ & $0(0,0 \%)$ \\
\hline & $60^{\circ}$ & $0 \quad(0,0 \%)$ & $0 \quad(0,0 \%)$ & $0(0,0 \%)$ \\
\hline
\end{tabular}

\begin{tabular}{|c|c|c|c|c|}
\hline \multirow[t]{2}{*}{ Grupo } & & \multicolumn{2}{|c|}{ Recidiva } & \multirow[t]{2}{*}{ Total } \\
\hline & & Presente & Ausente & \\
\hline \multirow[t]{2}{*}{ Primários } & G 1 & $9(52,9 \%)$ a $A$ & $8(47,1 \%) b A$ & 17 \\
\hline & G 2 & $3(27,3 \%)$ bA & $8(72,7 \%)$ a B & 11 \\
\hline \multirow[t]{2}{*}{ Recidivados } & G 1 & $6(75,0 \%)$ a B & $2(25,0 \%) b A$ & 8 \\
\hline & G 2 & $8(47,1 \%)$ bA & $9(52,9 \%)$ a A & 17 \\
\hline \multirow[t]{2}{*}{ Subtotal } & G 1 & $15(60,0 \%)$ a $A$ & $10(40,0 \%)$ b B & 25 \\
\hline & G 2 & $11(39,3 \%)$ b B & $17(60,7 \%)$ a $A$ & 28 \\
\hline Total & & $26(49,1 \%)$ & $27(50,9 \%)$ & 53 \\
\hline
\end{tabular}

Com a infiltração do $5 \mathrm{FU}$ pré-operatoriamente, não se observou alterações oculares em nenhum paciente.

Após o procedimento cirúrgico, em ambos os grupos estudados, observou-se a ocorrência de hiperemia, deiscência de sutura, além de ceratite e "cisto" conjuntival com "dellen" corneano.

A hiperemia é constante após o procedimento em todos os tipos de pterígio e técnicas cirúrgicas que sejam utilizadas para remoção da lesão. Regrediu mais rapidamente no G1, correspondente aos indivíduos que haviam recebido a infiltração do 5FU em época mais distante do momento cirúrgico, o que pode ter contribuído para o aparecimento menor do sinal.

Observou-se deiscência da sutura conjuntival em 14 casos $(26,4 \%)$ no $7^{\circ} \mathrm{PO}$. O 5FU, por ser droga antiproliferativa, pode diminuir ou retardar a cicatrização, de forma a propiciar a ocorrência de deiscências. Interessante notar que alguns indivíduos que tiveram deiscência da sutura apresentaram recidiva. Esta condição deve ser considerada como um fator facilitador para a progressão da lesão em direção ao limbo corneano.

Com relação às recidivas, observou-se que os indivíduos do G1 apresentaram índice de recidiva maior que os do G2.

Apesar das várias técnicas e tratamentos adjuvantes preconizados, as recidivas continuam a preocupar os cirurgiões. As recidivas podem estar associadas a diversos fatores, dentre eles: localização geográfica, ocupação do portador, idade, tipo de pterígio (involutivo ou carnoso; primário ou secundário), técnica cirúrgica empregada, uso de tratamentos coadjuvantes, tempo de seguimento(18). Procurou-se, então, avaliar se alguns destes fatores estariam associados aos índices de recidiva observados.
Com relação a isso, é importante valorizar o fato de que o G2 era constituído principalmente por portadores de pterígio recidivado e do tipo carnoso, lesões sabidamente mais agressivas e que, mesmo assim, apresentaram menor índice de recidiva.

A Mitomicina C, utilizada por injeção intra-lesional 30 dias antes da cirurgia, na concentração de $0,15 \mathrm{mg} / \mathrm{ml}$, foi considerada eficiente como tratamento coadjuvante do pterígio, com índice de $4 \%$ de recidiva em 9,2 meses de seguimento ${ }^{(14)}$.

Os indivíduos que receberam o 5FU 30 dias antes do procedimento (G1) apresentaram maiores índices de recidiva. O efeito do 5FU é mais transitório que o da Mitomicina $\mathrm{C}^{(19)}$. Este fato é relevante para determinar qual a melhor época para a aplicação da droga no pré-operatório, sendo necessária a aplicação em período inferior a 30 dias, o que vem corroborar com os melhores resultados observados no G2.

Já foi constatado, em cultura de células, que o efeito antifibroblástico do $5 \mathrm{FU}$ pode permanecer por até 36 dias após a administração da droga ${ }^{(19)}$. Porém, estudo feito em coelhos, com administração tópica de $5 \mathrm{FU}$ por 5 minutos, mostrou diminuição significativa do crescimento de fibroblastos em cultura de células entre 6 a 15 dias após a administração ${ }^{(20)}$.

Os menores índices de recidiva foram observados naqueles pacientes em que a aplicação do $5 \mathrm{FU}$ foi feita 10 dias pré-operatoriamente (G2). Porém, esta taxa de recidiva é maior que a observada com o uso da droga no intra-operatório ${ }^{(13)}$.

\section{CONCLUSÃO}

Portanto, não se observou nenhum paciente com complicações sérias em decorrência do uso do $5 \mathrm{FU}$ como injeção 
intra-lesional pré-operatoriamente. A aplicação de 5FU mostrouse mais efetiva quando feita 10 dias do que 30 dias antes do procedimento cirúrgico. Ainda assim, observou-se índice de recidiva superior ao encontrado com o uso intra-operatório. Outros estudos seriam necessários, visando estabelecer qual a época ideal para a utilização da medicação.

\section{ABS T RAC T}

Purpose: To assess the effect of 5-fluorouracil (5FU) injected in pterygia before surgery. Methods: 53 eyes of 52 patients ( 28 primary and 25 recurrent pterygia) underwent subconjunctival 5-fluorouracil application 30 days before surgery - group 1 (G1) and 10 days before surgery - group 2 (G2). All surgeries were performed by the same surgeon, who used the same technique in all patients. Patients were assessed 7, 30 and 60 days after surgery. Results were submitted to statistical analysis $(\mathrm{p}<0,05)$. Results: There were $52,8 \%$ primary and $47,2 \%$ recurrent pterygia, equally distributed regarding gender. There were no complications due to the infiltration of the drug. Recurrence occurred more frequently among recurrent pterygia and in G1. Conclusions: The use of subconjunctival 5-fluorouracil into the pterygium did not cause any deleterious effect to the studied eyes. There was less recurrence rate in the 10-day preoperative use of 5-fluorouracil when compared to the 30-day preoperative use of the drug.

Keywords: Pterygium/surgery; Ophthalmic solutions; Fluorouracil/administration \& dosage; Fluorouracil/therapeutic use; Mitomycin/therapeutic use; Recurrence/prevention \& control; Intraoperative care

\section{REFERÊNCIAS}

1. Alves MR. Contribuição ao estudo dos efeitos locais do tiotepa no pósoperatório do pterígio. Arq Bras Oftalmol 1990;53:203-9.
2. Alves MR, Gaiotto Junior OA, Kara-Jose N. Pterígio e tio-tepa. Arq Bras Oftalmol 1981;44:164-6.

3. Porto RB, Lacava AC, Falvo S, Centurion V. O uso da Mitomicina C tópica associada à exérese do pterígio. Rev Bras Oftalmol 1999;58:135-40.

4. Frucht-Perry J, Ilsan M, Hemo I. Single dosage of Mitomycin C for prevention of recurrent pterygium: preliminary report. Cornea 1994;13:411-3.

5. Yanyali AC, Talu H, Alp BN, Karabas L, Ay GM, Caglar Y. Intraoperative Mitomycin $C$ in the treatment of pterygium. Córnea 2000;19:471-3.

6. Rubinfeld RS, Pfister RR, Stein RM, Foster CS, Martin NF, Stoleru S, Talley $\mathrm{AR}$, et al. Serious complication of topical Mitomycin C after pterygium surgery [commented on Ophthalmology 1992;99:1645-6]. Ophthalmology 1992;99: 1647-54.

7. Fujitani A, Hayasaka S, Shibuia Y, Noda S. Corneoscleral ulceration and corneal perforation after pterygium excision and topical Mitomycin C therapy. Ophthalmologica 1993;207:162-4.

8. Cardilo JA, Alves MR, Ambrosio LA, Poterio MB, Kara-Jose N. Single intraoperative Mitomicin $\mathrm{C}$ eyedrops in pterygium surgery. Ophthalmology 1995; 102:1549-52.

9. Alves MR. Perda de ceratócitos após desepitelização da córnea e uso tópico de mitomicina C. Rev Bras Oftalmol 1996;55:268-75.

10. Cordova H, Castellanos R. Evaluación in vitro de la viabilidad y proliferación de fibroblastos de la cápsula de Tenon humana: expuestos a 5-fluorouracilo y Mitomicina C. Rev Oftalmol Venez 1997;53:12-21.

11. Barbarini LC, Schellini SA, Marques MEA. Efeitos da aplicação tópica de 5fluorouracil no limbo corneoescleral em coelhos [abstract]. Arq Bras Oftalmol 1999;62:409.

12. Komatsu F, Schellini SA, Marques MEA, Padovani CR, Camargo JLV. Efeitos da infiltração subconjuntival de 5-fluorouracil no limbo corneoescleral de coelhos [abstract]. Arq Bras Oftalmol 1999; 62:399.

13. Schellini SA, Shiratori CN, Spirandelli PH, Shiratori CA, Padovani CR. Uso do 5-fluorouracil no intra-operatório da cirurgia do pterígio. Arq Bras Oftalmol 2000;63:111-4

14. Donnenfield ED, Perry HD, Kornstein HS, Frommer SD, Kanellopoulos AI, D'Aversa G. Subconjuntival Mitomycin C as adjunctive therapy prior to pterygium excision [poster] In: American Academy of Ophthalmology; 1999 Oct 24-27, Orlando, EUA; 1999. p.155.

15. Pikkel J, Porges Y, Ophir A. Halting pterygium recurrence by postoperative 5- fluorouracil. Cornea 2001;20:168-71.

16. Goodman LA. Simultaneous confidence intervals for contrasts among multinomial populations. Annals Math Stat 1964;35(2):716-25.

17. Goodman LA. On Silmultaneous confidence intervals for multinomial proportions. Technometrics 1965;7:247-54.

18. Vasco Posada J. Pterígio: cirurgia y quimioterapia-curacion total, Medellin 1983-1988. CES Med 1989;3:85-9.

19. Khaw PT, Doyle JW, Sherwwod MB, Smith MF, Schultz G, McGorray S. Effects of intraoperative 5-fluouracil or mitomycin $\mathrm{C}$ on glaucoma filtration surgery in rabbit. Arch Ophthalmol 1993;100:367-75.

20. Khaw PT, Doyle JW, Sherwwod MB, Grierson I, Schultz G, McGorray S. Prolonged localized tissue effects from 5 minute exposures to fluorouracil and Mitomycin C. Arch Ophthalmol 1993;111:263-7.

\section{Ao enviar um artigo para publicação,}

leia ATENTAMENTE as instruções para autores,

constante no final de cada fascículo. 